\title{
Lie remarkable partial differential equations characterized by Lie algebras of point symmetries
}

\author{
Matteo Gorgone, Francesco Oliveri \\ Department MIFT, University of Messina \\ Viale F. Stagno d'Alcontres 31, 98166 Messina, Italy \\ mgorgone@unime.it; foliveri@unime.it \\ Published in J. Geom. Phys. 144, 314-323 (2019).
}

\begin{abstract}
Within the framework of inverse Lie problem, we give some nontrivial examples of coupled Lie remarkable equations, i.e., classes of differential equations that are in correspondence with their Lie point symmetries. In particular, we determine hierarchies of second order partial differential equations uniquely characterized by affine transformations of $\mathbb{R}^{n+m}$, and a system of two third order partial differential equations in two independent variables uniquely determined by the Lie algebra of projective transformations of $\mathbb{R}^{4}$.
\end{abstract}

Keywords. Lie symmetries; Jet spaces; Partial differential equations

Dedicated to Joseph Krasil'shchik on the occasion of his 70th birthday.

\section{Introduction}

Lie point symmetries of ordinary as well as partial differential equations are (finite or infinitesimal) transformations of the independent and dependent 
variables with the property of sending solutions into solutions [1, 2, 3, 4, 5. 6. 7, 8, ; they in turn induce transformations of the derivatives of the latter with respect to the former by requiring that the contact conditions are preserved. The set of all infinitesimal point symmetries, characterized by their infinitesimal generators, of a given differential equation span a vector space that has also the structure of a Lie algebra.

In Lie group analysis of differential equations, the direct problem of finding the admitted symmetries of a given differential equation can be complemented by a natural inverse problem, namely, the problem of finding the most general form of a differential equation (once the number of the independent and dependent variables and the maximal order of derivatives are fixed) admitting a given Lie algebra as subalgebra of its infinitesimal point symmetries.

There are different ways to consider this inverse problem. A first contribution aimed at characterizing all differential equations admitting a given symmetry group was given in [9]. In [10], the problem whether there exist non-trivial differential equations in one-to-one correspondence with their Lie point symmetries has been considered: the equation for the surfaces in $\mathbb{R}^{3}$ with vanishing Gaussian curvature has been recognized to be uniquely determined by its Lie point symmetries.

It is simple to check that many relevant differential equations can not be characterized by their Lie point symmetries since the dimension of the admitted Lie algebra is too small: KdV equation, Burgers' equation, Kepler's equations, ... are just some examples. An interesting contribution has been given by Rosenhaus in 1982 [10], who posed the problem of the unique determination of a differential equation by its group; in [10, the author considered the projective algebra of $\mathbb{R}^{3}$ and its subalgebras, and was able to prove that the equation of vanishing Gaussian curvature of surfaces in $\mathbb{R}^{3}$ (which is a Monge-Ampère-type equation) is uniquely determined by its Lie point symmetries. Moreover, in [11, 12], it was considered the problem of finding the minimal subalgebra of the algebra of point symmetries of the equation of vanishing Gaussian curvature of surfaces in $\mathbb{R}^{3}$ which uniquely determines it. Several authors studied the problem of finding, for a given equation, an extension of the algebra of point symmetries for which the equation at hand is determined. For instance, in [13] such a construction is performed in the case of ordinary differential equations by considering a non-local extension of the algebra of point symmetries, and the notion of complete symmetry group has been introduced (see also [14, 15, 16]). Also, in [10], it is shown that 
the algebra of contact symmetries is needed to characterize the equation of minimal surfaces.

The general approach to the inverse problem of constructing differential equations by using abstract Lie algebras requires to classify all possible realizations of the considered Lie algebra as algebra of vector fields on the space of independent and dependent variables. Then, looking for the differential invariants of the realization under consideration [17], under suitable hypotheses of regularity, the most general differential equation admitting a given Lie algebra as subalgebra of point symmetries is locally given as zeros of a set of smooth functions of the differential invariants.

The notion of Lie remarkable equation has been first introduced by one of the authors (F.O.) in [18, and used to analyze several classes of MongeAmpère equations [19, 20]. Then, in [21], the class of Lie remarkable equations, as determined by their Lie point symmetries, has been characterized within a geometrical framework, distinguishing them in weakly and strongly Lie remarkable equations; moreover, either necessary or sufficient conditions for a differential equation to be strongly or weakly Lie remarkable have been stated. Roughly speaking, strongly Lie remarkable equations are uniquely determined by their Lie point symmetries in the whole jet space, whereas weakly Lie remarkable equations are equations which do not intersect other equations admitting the same symmetries. Examples of Lie remarkable partial differential equations (Monge-Ampère equations, minimal surface equations) have been also given. Moreover, in [22, 23], differential equations uniquely determined by some relevant Lie algebras of vector fields in $\mathbb{R}^{3}$ have been characterized. Finally, in [24], the case of ordinary differential equations uniquely characterized by their Lie point symmetries has been investigated.

In this paper, we aim to determine Lie remarkable systems of partial differential equations, with special reference to the algebra of affine and projective transformations of $\mathbb{R}^{n+m}$, where $n \geq 2$ is the number of independent variables, and $m \geq 1$ the number of dependent ones.

The plan of the paper is as follows. In Section 2, we introduce a differential equation of order $r$ as a submanifold of a suitable jet space [3, 5] of order $r$. The symmetries are interpreted as particular vector fields on the jet space that are tangent to the differential equation. Then, we review the definitions of strongly and weakly Lie remarkable equations, and recall the main results obtained in [21]. The (strong or weak) Lie remarkability of differential equations is proved by computing the rank of the distributions determined by the prolongations of a Lie algebra and determining the submanifolds where 
the rank lowers. In Section 3, we identify a hierarchy of second order partial differential equations uniquely determined by the Lie algebra of affine transformations, whereas in Section 4, we present third order strongly Lie remarkable equations characterized by the Lie algebra of projective transformations. All the required computations have been carried out with the help of the Reduce [25] program ReLie [26]. Finally, Section 5 contains some concluding remarks.

\section{$2 \quad$ Theoretical setting}

In this Section, we recall some basic facts regarding Lie remarkable equations (see [21] for further details). The theory is carried out in the geometric framework of jet bundles [3, 5], assuming all manifolds and maps to be $C^{\infty}$.

Let $E$ be an $(n+m)$-dimensional smooth manifold. By using local charts of the form $\left(x_{i}, u_{\alpha}\right), i=1, \ldots, n, \alpha=1 \ldots m$, we describe (locally) an $n$ dimensional submanifold $L \subset E$ as the graph of a vector function $u_{\alpha}=$ $f_{\alpha}\left(x_{i}\right)$. In what follows, Latin indices run from 1 to $n$, and Greek indices run from 1 to $m$, unless otherwise specified. We denote by $\chi(E)$ the Lie algebra of vector fields on $E$.

The $r$-jet of $n$-dimensional submanifolds of $E$ (also known as extended jet bundles [3], or manifold of contact elements), $J^{r}(E, n)$, is the set of equivalence classes of submanifolds having at $p \in E$ a contact of order $r$. It has a smooth manifold structure with local charts $\left(x_{i}, u_{\alpha}, u_{\alpha, j_{1} \ldots j_{k}}\right)$, where

$$
u_{\alpha, j_{1} \ldots j_{k}} \equiv \frac{\partial^{k} u_{\alpha}}{\partial x_{j_{1}} \ldots \partial x_{j_{k}}}=\frac{\partial^{k} f_{\alpha}}{\partial x_{j_{1}} \ldots \partial x_{j_{k}}}, \quad 1 \leq k \leq r .
$$

It is

$$
\operatorname{dim}\left(J^{r}(E, n)\right)=n+m\left(\begin{array}{c}
n+r \\
r
\end{array}\right) .
$$

On $J^{r}(E, n)$ we have the contact distribution generated by the total derivative (Lie derivative)

$$
\frac{D}{D x_{i}} \stackrel{\text { def }}{=} \frac{\partial}{\partial x_{i}}+\sum_{\alpha=1}^{m}\left(u_{\alpha, i} \frac{\partial}{\partial u_{\alpha}}+\sum_{k=1}^{r-1} \sum_{j_{1}=1}^{n} \ldots \sum_{j_{k}=1}^{n} u_{\alpha, j_{1} \ldots j_{k} i} \frac{\partial}{\partial u_{\alpha, j_{1} \ldots j_{k}}}\right) .
$$


Any vector field $\Xi \in \chi(E)$,

$$
\Xi=\sum_{i=1}^{n} \xi_{i}\left(x_{j}, u_{\beta}\right) \frac{\partial}{\partial x_{i}}+\sum_{\alpha=1}^{m} \eta_{\alpha}\left(x_{j}, u_{\beta}\right) \frac{\partial}{\partial u_{\alpha}},
$$

can be lifted to a vector field $\Xi^{(r)} \in \chi\left(J^{r}(E, n)\right)$,

$$
\Xi^{(r)}=\sum_{i=1}^{n} \xi_{i} \frac{\partial}{\partial x_{i}}+\sum_{\alpha=1}^{m}\left(\eta_{\alpha} \frac{\partial}{\partial u_{\alpha}}+\sum_{k=1}^{r} \sum_{j_{1}=1}^{n} \ldots \sum_{j_{k}=1}^{n} \eta_{\left[\alpha, j_{1} \ldots j_{k}\right]} \frac{\partial}{\partial u_{\alpha, j_{1} \ldots j_{k}}}\right),
$$

where

$$
\eta_{\left[\alpha, j_{1} \ldots j_{k}\right]}=\frac{D \eta_{\left[\alpha, j_{1} \ldots j_{k-1}\right]}}{D x_{j_{k}}}-\sum_{\ell=1}^{n} \frac{D \xi_{\ell}}{D x_{j_{k}}} u_{\alpha, j_{1} \ldots j_{k-1} \ell}, \quad \eta_{[\alpha]}=\eta_{\alpha} .
$$

A differential equation $\mathcal{E}$ of order $r$ on an $n$-dimensional submanifold of a manifold $E$ is a submanifold of $J^{r}(E, n)$. An infinitesimal point symmetry of $\mathcal{E}$ is a vector field of the type $\Xi^{(r)}$ which is tangent to $\mathcal{E}$. If $\mathcal{E}$ is locally described by

$$
\Delta_{i}=0, \quad i=1, \ldots, q<\operatorname{dim}\left(J^{r}(E, n)\right),
$$

where

$$
\Delta_{i}: J^{r}(E, n) \rightarrow \mathbb{R},
$$

then point symmetries are the solutions of the system

$$
\left.\Xi^{(r)}\left(\Delta_{i}\right)\right|_{\Delta_{1}=\cdots=\Delta_{q}=0}=0, \quad i=1, \ldots, q .
$$

We denote by $\operatorname{sym}(\mathcal{E})$ the Lie algebra of infinitesimal point symmetries of the equation $\mathcal{E}$.

The problem of determining the Lie algebra $\operatorname{sym}(\mathcal{E})$ is said to be the direct Lie problem. Conversely, given a Lie subalgebra $\mathfrak{S} \subset \chi\left(J^{r}(E, n)\right)$ of contact vector fields, we consider the inverse Lie problem, i.e., the problem of classifying the equations $\mathcal{E} \subset J^{r}(E, n)$ such that $\operatorname{sym}(\mathcal{E}) \supseteq \mathfrak{S}$.

For the reader's convenience, let us now recall the definitions and the main properties, contained in [21], of differential equations which are characterized by their Lie point symmetries, that we call Lie remarkable. 
Definition 1 (Weakly Lie remarkable equations) Let $E$ be a manifold, $\operatorname{dim}(E)=n+m$, and let $r \in \mathbb{N}, r>0$. An $\ell$-dimensional equation $\mathcal{E} \subset$ $J^{r}(E, n)$ is said to be weakly Lie remarkable with respect to a Lie algebra $\mathfrak{S}$ of point symmetries if $\mathcal{E}$ is the only maximal (with respect to the inclusion) $\ell$-dimensional equation in $J^{r}(E, n)$ passing at any $\theta \in \mathcal{E}$ and admitting $\mathfrak{S}$ as a subalgebra of its infinitesimal point symmetries.

Definition 2 (Strongly Lie remarkable equations) Let $E$ be a manifold, $\operatorname{dim}(E)=n+m$, and let $r \in \mathbb{N}, r>0$. An $\ell$-dimensional equation $\mathcal{E} \subset J^{r}(E, n)$ is said to be strongly Lie remarkable with respect to a Lie algebra $\mathfrak{S}$ of point symmetries if $\mathcal{E}$ is the only maximal (with respect to the inclusion) $\ell$-dimensional equation in $J^{r}(E, n)$ admitting $\mathfrak{S}$ as a subalgebra of its infinitesimal point symmetries.

Of course, a strongly Lie remarkable equation is also weakly Lie remarkable.

Some direct consequences of the above definitions are in order. For each $\theta \in J^{r}(E, n)$, let us denote by $S_{\theta}(\mathcal{E}) \subset T_{\theta} J^{r}(E, n)$ the subspace generated by the values of infinitesimal point symmetries of $\mathcal{E}$ at $\theta$. Let us set

$$
S(\mathcal{E}) \stackrel{\text { def }}{=} \bigcup_{\theta \in J^{r}(E, n)} S_{\theta}(\mathcal{E}) .
$$

In general, $\operatorname{dim}\left(S_{\theta}(\mathcal{E})\right)$ may change with $\theta \in J^{r}(E, n)$. It is clear that $\operatorname{dim}(\operatorname{sym}(\mathcal{E})) \geq \operatorname{dim}\left(S_{\theta}(\mathcal{E})\right)$, for all $\theta \in J^{r}(E, n)$. If the rank of $S(\mathcal{E})$ at each $\theta \in J^{r}(E, n)$ equals $\operatorname{dim}(\operatorname{sym}(\mathcal{E}))$, then $S(\mathcal{E})$ is an involutive (smooth) distribution. A submanifold $N$ of $J^{r}(E, n)$ is an integral submanifold of $S(\mathcal{E})$ if $T_{\theta} N=S_{\theta}(\mathcal{E})$ for each $\theta \in N$. Of course, an integral submanifold of $S(\mathcal{E})$ is an equation in $J^{r}(E, n)$ which admits all elements in $\operatorname{sym}(\mathcal{E})$ as infinitesimal point symmetries. Moreover, due to the fact that point symmetries of $\mathcal{E}$ are tangent to $\mathcal{E}$, we have $\operatorname{dim}\left(S_{\theta}(\mathcal{E})\right) \leq \ell$.

The points of $J^{r}(E, n)$ of maximal rank of $S(\mathcal{E})$ form an open set of $J^{r}(E, n)$ (see [21]). It follows that $\mathcal{E}$ can not coincide with the set of points of maximal rank of $S(\mathcal{E})$.

In [21], the following results, concerned with either necessary or sufficient conditions for a differential equation to be weakly or strongly Lie remarkable, have been proved.

Theorem 1 A necessary condition for the differential equation $\mathcal{E}$ to be strongly Lie remarkable is that $\operatorname{dim}(\operatorname{sym}(\mathcal{E}))>\operatorname{dim}(\mathcal{E})$. 
Theorem 2 A necessary condition for the differential equation $\mathcal{E}$ to be weakly Lie remarkable is that $\operatorname{dim}(\operatorname{sym}(\mathcal{E})) \geq \operatorname{dim}(\mathcal{E})$.

Theorem 3 If $\left.S(\mathcal{E})\right|_{\mathcal{E}}$ is an $\ell$-dimensional distribution on $\mathcal{E} \subset J^{r}(E, n)$, then $\mathcal{E}$ is a weakly Lie remarkable equation.

Theorem 4 Let $S(\mathcal{E})$ be such that for any $\theta \notin \mathcal{E}$ we have $\operatorname{dim}\left(S_{\theta}(\mathcal{E})\right)>\ell$. Then $\mathcal{E}$ is a strongly Lie remarkable equation.

By using these definitions and results, in the next sections we will determine some classes of new Lie remarkable differential equations.

\section{Second order Lie remarkable equations}

The Lie algebra $\mathcal{A}\left(\mathbb{R}^{n+m}\right)$ of affine transformations of $\mathbb{R}^{n+m}$ is spanned by the vector fields

$$
\frac{\partial}{\partial a}, \quad a \frac{\partial}{\partial b}
$$

where $a, b \in\left\{x_{1}, \ldots, x_{n}, u_{1}, \ldots, u_{m}\right\}$; it is

$$
\operatorname{dim}\left(\mathcal{A}\left(\mathbb{R}^{n+m}\right)\right)=(m+n)(m+n+1) ;
$$

on the contrary, as far as the second order jet space is concerned, it is

$$
\operatorname{dim}\left(J^{2}\left(\mathbb{R}^{n+m}, n\right)\right)=n+m(n+1)(n+2) / 2,
$$

whereupon it is immediate to prove that

$$
\operatorname{dim}\left(\mathcal{A}\left(\mathbb{R}^{n+m}\right)\right)>\operatorname{dim}\left(J^{2}\left(\mathbb{R}^{n+m}, n\right)\right)
$$

for all $m$ provided that $n \leq 5$. Nevertheless, in order to characterize strongly (weakly, respectively) Lie remarkable equations, it is necessary that the dimension of the Lie algebra be greater (greater or equal, respectively) to the dimension of the equation. In the following, we shall prove that strongly Lie remarkable equations corresponding to the Lie algebra of affine transformations of $\mathbb{R}^{n+m}$ exist for all values of $m$ and $n$.

In [21], it was proved that for $n=2, m=1$, the Monge-Ampère equation for a surface with vanishing Gaussian curvature,

$$
u_{, 11} u_{, 22}-u_{, 12}^{2}=0,
$$


is strongly Lie remarkable: equation (1) is uniquely characterized by the Lie algebra of affine transformations, even if it admits the Lie algebra of projective transformations of $\mathbb{R}^{3}$.

This result has been extended [21] to $n$-dimensional manifolds in $\mathbb{R}^{n+1}$, as stated by the next theorem.

Theorem 5 The unique second order partial differential equation for the unknown $u\left(x_{1}, \ldots, x_{n}\right)(n \geq 2)$, characterized by the Lie algebra of affine transformations of $\mathbb{R}^{n+1}$, is

$$
\operatorname{det}\left\|\begin{array}{llll}
u_{, 11} & u_{, 12} & \ldots & u_{, 1 n} \\
u_{, 12} & u_{, 22} & \ldots & u_{, 2 n} \\
\cdots & \ldots & \ldots & \ldots \\
u_{, 1 n} & u_{, 2 n} & \ldots & u_{, n n}
\end{array}\right\|=0 \text {, }
$$

that so is strongly Lie remarkable.

Proof. The second order prolongation of vector fields of Lie algebra of affine transformations of $\mathbb{R}^{n+1}$ gives a distribution of maximal rank (equal to $(n+1)(n+2))$; the only maximal manifold where this rank lowers to $(n+1)(n+2)-1$ is characterized by the local expression (2).

The Monge-Ampère equation for a surface with vanishing Gaussian curvature is a particular instance of the equation introduced by Ampère in a paper published in 1815 on the Journal de l'École Polytechnique [27], having the form

$$
H \frac{\partial^{2} u}{\partial t^{2}}+2 K \frac{\partial^{2} u}{\partial t \partial x}+L \frac{\partial^{2} u}{\partial x^{2}}+M+N\left(\frac{\partial^{2} u}{\partial t^{2}} \frac{\partial^{2} u}{\partial x^{2}}-\left(\frac{\partial^{2} u}{\partial t \partial x}\right)^{2}\right)=0
$$

where the coefficients $H, K, L, M, N \neq 0$ depend on $t, x, u$ and first order derivatives. A remarkable property of equation (3) has been proved by Boillat [28], who showed that (3) is the most general second order nonlinear hyperbolic equation in $(1+1)$ dimensions that is completely exceptional. In 1973, Ruggeri [29], using the property of complete exceptionality, generalized the Monge-Ampère equation to $(2+1)$ dimensions. Moreover, Donato, Ramgulam, and Rogers [30] characterized the $(3+1)$-dimensional MongeAmpère equation. Boillat [31, with the same procedure, derived the general form of Monge-Ampère equation for a function $u\left(x_{1}, \ldots, x_{n}\right)$. All these second order Monge-Ampère equations are given as a linear combination (with 
coefficients arbitrary functions of the independent variables, the dependent variable and its first order derivatives) of all minors of all orders extracted from the Hessian matrix. Some of these equations can be reduced to quasilinear or linear form [32, 33, 34]. The property of complete exceptionality has been used by Boillat [35] to characterize higher order Monge-Ampère equations for a function $u\left(x_{1}, x_{2}\right)$; in such a case, they are given as a linear combination of all minors of all orders extracted from the Hankel matrix.

Now, we consider the situation where $m>1$, i.e., the case of a system of partial differential equations, and derive new strongly Lie remarkable equations.

Theorem 6 The system of second order partial differential equations locally described in $J^{2}\left(\mathbb{R}^{4}, 2\right)$ by

$$
\left\{\begin{array}{l}
u_{1,11} u_{2,12}-u_{1,12} u_{2,11}=0, \\
u_{1,11} u_{2,22}-u_{1,22} u_{2,11}=0,
\end{array}\right.
$$

is strongly Lie remarkable.

Proof. System (4) characterizes a 12-dimensional manifold in the second order jet space $J^{2}\left(\mathbb{R}^{4}, 2\right)$, that has dimension 14 . The dimension of the Lie algebra of affine transformations of $\mathbb{R}^{4}$ is 20 , and, by a straightforward calculation, one can easily recognize that the rank of the distribution of its second order prolongation is 14 , reducing to 12 only on the 12-dimensional manifold of $J^{2}\left(\mathbb{R}^{4}, 2\right)$ characterized by (44); therefore, system (4) is strongly Lie remarkable. In passing, we note that system (4) admits also the symmetries generated by

$$
a\left(x_{1} \frac{\partial}{\partial x_{1}}+x_{2} \frac{\partial}{\partial x_{2}}+u_{1} \frac{\partial}{\partial u_{1}}+u_{2} \frac{\partial}{\partial u_{2}}\right),
$$

where $a \in\left\{x_{1}, x_{2}, u_{1}, u_{2}\right\}$, i.e., it admits the 24-dimensional Lie algebra of projective transformations of $\mathbb{R}^{4}$.

Remark 1 In a 4-dimensional Euclidean space, the functions

$$
u_{1}=u_{1}\left(x_{1}, x_{2}\right), \quad u_{2}=u_{2}\left(x_{1}, x_{2}\right)
$$

characterize a 2-dimensional surface. In [36], the authors introduced an invariant linear map of Weingarten type in the tangent space of the surface, 
which generates two invariants $k$ and $\kappa$. These invariants divide the points of the surface into 4 types: flat, elliptic, parabolic and hyperbolic. The two invariants (see [36] for the details) for the surface defined by (6) have the form

$$
\begin{aligned}
k & =\frac{4\left(u_{1,11} u_{2,12}-u_{1,12} u_{2,11}\right)\left(u_{1,12} u_{2,22}-u_{1,22} u_{2,12}\right)-\left(u_{1,11} u_{2,22}-u_{1,22} u_{2,11}\right)^{2}}{\left(1+u_{1,1}^{2}+u_{1,2}^{2}+u_{2,1}^{2}+u_{2,2}^{2}+\left(u_{1,1} u_{2,2}+u_{1,2} u_{2,1}\right)^{2}\right)^{3}}, \\
\kappa & =\frac{\left(1+u_{1,1}^{2}+u_{2,1}^{2}\right)\left(u_{1,12} u_{2,22}-u_{1,22} u_{2,12}\right)}{\left(1+u_{1,1}^{2}+u_{1,2}^{2}+u_{2,1}^{2}+u_{2,2}^{2}+\left(u_{1,1} u_{2,2}+u_{1,2} u_{2,1}\right)^{2}\right)^{2}} \\
& +\frac{\left(1+u_{1,2}^{2}+u_{2,2}^{2}\right)\left(u_{1,11} u_{2,12}-u_{1,12} u_{2,11}\right)}{\left(1+u_{1,1}^{2}+u_{1,2}^{2}+u_{2,1}^{2}+u_{2,2}^{2}+\left(u_{1,1} u_{2,2}+u_{1,2} u_{2,1}\right)^{2}\right)^{2}} \\
& -\frac{\left(u_{1,1} u_{1,2}-u_{2,1} u_{2,2}\right)\left(u_{1,11} u_{2,22}-u_{1,22} u_{2,11}\right)}{\left(1+u_{1,1}^{2}+u_{1,2}^{2}+u_{2,1}^{2}+u_{2,2}^{2}+\left(u_{1,1} u_{2,2}+u_{1,2} u_{2,1}\right)^{2}\right)^{2}} .
\end{aligned}
$$

It is immediately seen that both invariants vanish identically on the equations (41), whereupon it remains proved that these equations characterize the surfaces consisting of flat points: such surfaces are either planar surfaces or developable ruled surfaces [36].

Second order strongly Lie remarkable systems do exist also for $m>2$ or $n>2$. Before stating the theorem that identifies such systems for arbitrary values of $m$ and $n$, let us consider some special cases.

Theorem 7 The system of second order partial differential equations locally described in $J^{2}\left(\mathbb{R}^{5}, 3\right)$ by

$$
\left\{\begin{array}{l}
u_{1,11} u_{2,12}-u_{1,12} u_{2,11}=0 \\
u_{1,11} u_{2,13}-u_{1,13} u_{2,11}=0 \\
u_{1,11} u_{2,22}-u_{1,22} u_{2,11}=0 \\
u_{1,11} u_{2,23}-u_{1,23} u_{2,11}=0 \\
u_{1,11} u_{2,33}-u_{1,33} u_{2,11}=0
\end{array}\right.
$$

is strongly Lie remarkable.

Proof. System (7) characterizes a 18-dimensional manifold in the second order jet space $J^{2}\left(\mathbb{R}^{5}, 3\right)$, that has dimension 23 . The dimension of the Lie algebra of affine transformations of $\mathbb{R}^{5}$ is 30 , and, by a straightforward 
calculation, one can easily recognize that the rank of the distribution of its second order prolongation is 23, reducing to 18 only on the 18-dimensional manifold of $J^{2}\left(\mathbb{R}^{5}, 3\right)$ characterized by (7), that, consequently, is strongly Lie remarkable. Actually, the system (17) admits the 35-dimensional Lie algebra of projective transformations of $\mathbb{R}^{5}$.

Theorem 8 The system of second order partial differential equations locally described in $J^{2}\left(\mathbb{R}^{5}, 2\right)$ by

$$
\left\{\begin{array}{l}
u_{1,11} u_{2,12}-u_{1,12} u_{2,11}=0 \\
u_{1,11} u_{2,22}-u_{1,22} u_{2,11}=0 \\
u_{2,11} u_{3,12}-u_{2,12} u_{3,11}=0 \\
u_{2,11} u_{3,22}-u_{2,22} u_{3,11}=0
\end{array}\right.
$$

is strongly Lie remarkable.

Proof. System (8) characterizes a 16-dimensional manifold in the second order jet space $J^{2}\left(\mathbb{R}^{5}, 2\right)$, that has dimension 20 . The dimension of the Lie algebra of affine transformations of $\mathbb{R}^{5}$ is 30 , and, by a straightforward calculation, one can easily recognize that the rank of the distribution of its second order prolongation is 20, reducing to 16 only on the 16-dimensional manifold of $J^{2}\left(\mathbb{R}^{5}, 2\right)$ characterized by (8) , that, consequently, is strongly Lie remarkable. Actually, it is easily verified that system (8) admits the 35-dimensional Lie algebra of projective transformations of $\mathbb{R}^{5}$ too.

For arbitrary values of $m$ and $n$, we are able to state the following result.

Theorem 9 The system of second order partial differential equations in the unknowns $\left(u_{1}\left(x_{1}, \ldots, x_{n}\right), \ldots, u_{m}\left(x_{1}, \ldots, x_{n}\right)\right)$

$$
\Delta=\mathbf{0}
$$

with the components of $\boldsymbol{\Delta}$ given by

$$
\Delta_{\alpha, \alpha+1 ; 1,1, p, q} \equiv u_{\alpha, 11} u_{\alpha+1, p q}-u_{\alpha, p q} u_{\alpha+1,11}=0,
$$

where $\alpha=1, \ldots, m-1$ and $p, q=1, \ldots, n$, is strongly Lie remarkable.

Proof. System (9), made by $(m-1)\left(n^{2}+n-2\right) / 2$ independent differential equations, characterizes a manifold with dimension $d$ in the second order jet space $J^{2}\left(\mathbb{R}^{n+m}, n\right)$, where

$$
d=n^{2}+2 n+3 m-2+\frac{m n(1-n)}{2} .
$$


A simple analysis shows that $d<(n+m)(n+m+1)$ for all positive values of $m$ and $n$, so that the number of symmetries in the affine Lie algebra of $\mathbb{R}^{n+m}$, according to Theorem 4, may suffice. Moreover, the rank of the distribution of the second order prolongation of the generators of affine Lie algebra in $\mathbb{R}^{n+m}$ is maximal (the minimum between the dimension of affine Lie algebra and the dimension of jet space).

It is easy to recognize that from (10) it follows

$$
\Delta_{\alpha, \beta ; i, j, p, q} \equiv u_{\alpha, i j} u_{\beta, p q}-u_{\alpha, p q} u_{\beta+1, i j}=0,
$$

for all $\alpha, \beta \in\{1, \ldots, m\}$ and all $i, j, p, q \in\{1, \ldots, n\}$.

Now, let us prove that system (9) admits as subalgebra of the algebra of its Lie point symmetries the Lie algebra of affine transformations. In the sequel, we use the Einstein summation convention over repeated indices.

Consider the infinitesimal generators of the affine Lie algebra in $\mathbb{R}^{n+m}$, say

$$
\frac{\partial}{\partial x_{i}}, \quad \frac{\partial}{\partial u_{\beta}}, \quad x_{j} \frac{\partial}{\partial x_{i}}, \quad u_{\beta} \frac{\partial}{\partial x_{i}}, \quad x_{i} \frac{\partial}{\partial u_{\beta}}, \quad u_{\gamma} \frac{\partial}{\partial u_{\beta}},
$$

where $i, j=1, \ldots, n$ and $\beta, \gamma=1, \ldots, m$. We will prove that each of the vector fields in (12) is admitted by system (9).

Since neither the independent variables nor the dependent ones appear explicitly in system (9), it is evident the invariance with respect to the $(n+m)$ translations of the independent and dependent variables. Let us consider the remaining vector fields.

1. $\Xi=x_{j} \frac{\partial}{\partial x_{i}}$. Its second order prolongation is

$$
\Xi^{(2)}=x_{j} \frac{\partial}{\partial x_{i}}-\delta_{j, k} u_{\gamma, i} \frac{\partial}{\partial u_{\gamma, k}}-\left(\delta_{j, \ell} u_{\gamma, i k}+\delta_{j, k} u_{\gamma, i \ell}\right) \frac{\partial}{\partial u_{\gamma, k \ell}},
$$

where $\delta_{i, j}$ is the Kronecker symbol. It is:

$$
\begin{aligned}
& \Xi^{(2)}\left(\Delta_{\alpha, \alpha+1 ; 1,1, p, q}\right)= \\
& \quad=2 \delta_{j, 1}\left(u_{\alpha, 1 i} u_{\alpha+1, p q}-u_{\alpha, p q} u_{\alpha+1,1 i}\right) \\
& +\delta_{j, p}\left(u_{\alpha, 11} u_{\alpha+1, i q}-u_{\alpha, i q} u_{\alpha+1,11}\right) \\
& +\delta_{j, q}\left(u_{\alpha, 11} u_{\alpha+1, i p}-u_{\alpha, i p} u_{\alpha+1,11}\right) \\
& =2 \delta_{j, 1} \Delta_{\alpha, \alpha+1 ; 1, i, p, q}+\delta_{j, p} \Delta_{\alpha, \alpha+1 ; 1,1, i, q}+\delta_{j, q} \Delta_{\alpha, \alpha+1 ; 1,1, i, p},
\end{aligned}
$$

that vanishes on $\boldsymbol{\Delta}=\mathbf{0}$; 
2. $\Xi=u_{\beta} \frac{\partial}{\partial x_{i}}$. Its second order prolongation is

$$
\begin{aligned}
\Xi^{(2)} & =u_{\beta} \frac{\partial}{\partial x_{i}}-u_{\gamma, i} u_{\beta, k} \frac{\partial}{\partial u_{\gamma, k}} \\
& -\left(u_{\gamma, i} u_{\beta, k \ell}+u_{\beta, k} u_{\gamma, i \ell}+u_{\beta, \ell} u_{\gamma, i k}\right) \frac{\partial}{\partial u_{\gamma, k \ell}}
\end{aligned}
$$

whereupon

$$
\begin{aligned}
& \Xi^{(2)}\left(\Delta_{\alpha, \alpha+1 ; 1,1, p, q}\right)= \\
& =u_{\alpha, i}\left(u_{\beta, 11} u_{\alpha+1, p q}-u_{\beta, p q} u_{\alpha+1,11}\right) \\
& +u_{\alpha+1, i}\left(u_{\alpha, 11} u_{\beta, p q}-u_{\alpha, p q} u_{\beta, 11}\right) \\
& +2 u_{\beta, 1}\left(u_{\alpha, 1 i} u_{\alpha+1, p q}-u_{\alpha, p q} u_{\alpha+1,1 i}\right) \\
& +u_{\beta, p}\left(u_{\alpha, 11} u_{\alpha+1, i q}-u_{\alpha, i q} u_{\alpha+1,11}\right) \\
& +u_{\beta, q}\left(u_{\alpha, 11} u_{\alpha+1, i p}-u_{\alpha, i p} u_{\alpha+1,11}\right) \\
& =u_{\alpha, i} \Delta_{\beta, \alpha+1 ; 1,1, p, q}+u_{\alpha+1, i} \Delta_{\alpha, \beta ; 1,1, p, q} \\
& +2 u_{\beta, 1} \Delta_{\alpha, \alpha+1 ; 1, i, p, q}+u_{\beta, p} \Delta_{\alpha, \alpha+1 ; 1,1, i, q}+u_{\beta, q} \Delta_{\alpha, \alpha+1 ; 1,1, i, p}
\end{aligned}
$$

vanishing on $\boldsymbol{\Delta}=\mathbf{0}$;

3. $\Xi=x_{i} \frac{\partial}{\partial u_{\beta}}$. Its second order prolongation reads

$$
\Xi^{(2)}=x_{i} \frac{\partial}{\partial u_{\beta}}+\delta_{\beta, \gamma} \delta_{i, k} \frac{\partial}{\partial u_{\gamma, k}}
$$

whereupon

$$
\Xi^{(2)}\left(\Delta_{\alpha, \alpha+1 ; 1,1, p, q}\right)=0 ;
$$

4. $\Xi=u_{\gamma} \frac{\partial}{\partial u_{\beta}}$. Its second order prolongation is

$$
\Xi^{(2)}=u_{\gamma} \frac{\partial}{\partial u_{\beta}}+\delta_{\beta, \mu} u_{\gamma, k} \frac{\partial}{\partial u_{\mu, k}}+\delta_{\beta, \mu} u_{\gamma, k \ell} \frac{\partial}{\partial u_{\mu, k \ell}},
$$

whereupon

$$
\begin{aligned}
& \Xi^{(2)}\left(\Delta_{\alpha, \alpha+1 ; 1,1, p, q}\right)= \\
& =\delta_{\alpha, \beta}\left(u_{\gamma, 11} u_{\alpha+1, p q}-u_{\gamma, p q} u_{\alpha+1,11}\right) \\
& +\delta_{\alpha+1, \beta}\left(u_{\alpha, 11} u_{\gamma, p q}-u_{\alpha, p q} u_{\gamma, 11}\right) \\
& =\delta_{\alpha, \beta} \Delta_{\gamma, \alpha+1 ; 1,1, p, q}+\delta_{\alpha+1, \beta} \Delta_{\alpha, \gamma ; 1,1, p, q}
\end{aligned}
$$

vanishing on $\boldsymbol{\Delta}=\mathbf{0}$, so completing the proof. 
We also observe that system (9) is invariant with respect to the projective transformations of $\mathbb{R}^{n+m}$ too.

Proposition 1 System (91) admits the symmetries generated by the vector fields

$$
a\left(\sum_{i=1}^{n} x_{i} \frac{\partial}{\partial x_{i}}+\sum_{\alpha=1}^{m} u_{\alpha} \frac{\partial}{\partial u_{\alpha}}\right)
$$

with $a \in\left\{x_{1}, \ldots, x_{n}, u_{1}, \ldots, u_{m}\right\}$, so that system (9) is strongly Lie remarkable also with respect to the Lie algebra of projective transformations of $\mathbb{R}^{n+m}$.

Proof. We need to prove that system (9) admits also the symmetries spanned by the $(n+m)$ vector fields

$$
x_{j}\left(\sum_{i=1}^{n} x_{i} \frac{\partial}{\partial x_{i}}+\sum_{\gamma=1}^{m} u_{\gamma} \frac{\partial}{\partial u_{\gamma}}\right), \quad u_{\beta}\left(\sum_{i=1}^{n} x_{i} \frac{\partial}{\partial x_{i}}+\sum_{\gamma=1}^{m} u_{\gamma} \frac{\partial}{\partial u_{\gamma}}\right),
$$

where $j=1, \ldots, n$, and $\beta=1, \ldots, m$.

1. $\Xi=x_{j}\left(x_{i} \frac{\partial}{\partial x_{i}}+u_{\gamma} \frac{\partial}{\partial u_{\gamma}}\right)$ (sum over repeated indices).

Its second order prolongation is

$$
\begin{aligned}
\Xi^{(2)} & =x_{j} x_{i} \frac{\partial}{\partial x_{i}}+x_{j} u_{\gamma} \frac{\partial}{\partial u_{\gamma}}+\delta_{j, k}\left(u_{\gamma}-x_{m} u_{\gamma, m}\right) \frac{\partial}{\partial u_{\gamma, k}} \\
& -\left(\delta_{j, k} u_{\gamma, m} u_{\gamma, \ell m}+\delta_{j, \ell} x_{m} u_{\gamma, k m}+x_{j} u_{\gamma, k \ell}\right) \frac{\partial}{\partial u_{\gamma, k \ell}} .
\end{aligned}
$$

It is

$$
\begin{aligned}
& \Xi^{(2)}\left(\Delta_{\alpha, \alpha+1 ; 1,1, p, q}\right)= \\
& =\delta_{j, p} x_{m}\left(u_{\alpha, m q} u_{\alpha+1,11}-u_{\alpha, 11} u_{\alpha+1, m q}\right) \\
& +\delta_{j, q} x_{m}\left(u_{\alpha, m p} u_{\alpha+1,11}-u_{\alpha, 11} u_{\alpha+1, m p}\right) \\
& +2 \delta_{j, 1} x_{m}\left(u_{\alpha, p q} u_{\alpha+1,1 m}-u_{\alpha, 1 m} u_{\alpha+1, p q}\right) \\
& +2 x_{j}\left(u_{\alpha, p q} u_{\alpha+1,11}-u_{\alpha, 11} u_{\alpha+1, p q}\right) \\
& =-x_{m}\left(\delta_{j, p} \Delta_{\alpha, \alpha+1 ; 1,1, m, q} \delta_{j, q}+\Delta_{\alpha, \alpha+1 ; 1,1, m, p}+2 \delta_{j, 1} \Delta_{\alpha, \alpha+1 ; 1, m, p, q}\right) \\
& -2 x_{j} \Delta_{\alpha, \alpha+1 ; 1,1, p, q}
\end{aligned}
$$

that vanishes on $\boldsymbol{\Delta}=\mathbf{0}$; 
2. $\Xi=u_{\beta}\left(x_{i} \frac{\partial}{\partial x_{i}}+u_{\gamma} \frac{\partial}{\partial u_{\gamma}}\right)$ (sum over repeated indices).

Its second order prolongation is

$$
\begin{aligned}
\Xi^{(2)} & =u_{\beta} x_{i} \frac{\partial}{\partial x_{i}}+u_{\beta} u_{\gamma} \frac{\partial}{\partial u_{\gamma}}+u_{\beta, k}\left(u_{\gamma}-x_{i} u_{\gamma, i}\right) \frac{\partial}{\partial u_{\gamma, k}} \\
& +\left(u_{\gamma} u_{\beta, k \ell}-u_{\beta} u_{\gamma, k \ell}\right. \\
& \left.-x_{i}\left(u_{\gamma, i} u_{\beta, k \ell}+u_{\beta, k} u_{\gamma, i \ell}+u_{\beta, l} u_{\gamma, i k}\right)\right) \frac{\partial}{\partial u_{\gamma, k \ell}} .
\end{aligned}
$$

It is

$$
\begin{aligned}
& \Xi^{(2)}\left(\Delta_{\alpha, \alpha+1 ; 1,1, p, q}\right)= \\
& =\left(u_{\alpha}-x_{i} u_{\alpha, i}\right)\left(u_{\gamma, 11} u_{\alpha+1, p q}-u_{\gamma, p q} u_{\alpha+1,11}\right) \\
& +\left(u_{\alpha+1}-x_{i} u_{\alpha+1, i}\right)\left(u_{\alpha, 11} u_{\gamma, p q}-u_{\alpha, p q} u_{\gamma, 11}\right) \\
& -2 u_{\gamma}\left(u_{\alpha, 11} u_{\alpha+1, p q}-u_{\alpha, p q} u_{\alpha+1,11}\right) \\
& -2 x_{i} u_{\gamma, 1}\left(u_{\alpha, 1 i} u_{\alpha+1, p q}-u_{\alpha, p q} u_{\alpha+1,1 i}\right) \\
& -x_{i} u_{\gamma, p}\left(u_{\alpha, 11} u_{\alpha+1, i q}-u_{\alpha, i q} u_{\alpha+1,11}\right) \\
& -x_{i} u_{\gamma, q}\left(u_{\alpha, 11} u_{\alpha+1, i p}-u_{\alpha, i p} u_{\alpha+1,11}\right) \\
& =\left(u_{\alpha}-x_{i} u_{\alpha, i} \Delta_{\gamma, \alpha+1 ; 1,1, p, q}+\left(u_{\alpha+1}-x_{i} u_{\alpha+1, i}\right) \Delta_{\alpha, \gamma ; 1,1, p, q}\right. \\
& -2 u_{\gamma} \Delta_{\alpha, \alpha+1 ; 1,1, p, q}-2 x_{i} u_{\gamma, 1} \Delta_{\alpha, \alpha+1 ; 1, i, p, q} \\
& -x_{i} u_{\gamma, p} \Delta_{\alpha, \alpha+1 ; 1,1, i, q}-x_{i} u_{\gamma, q} \Delta_{\alpha, \alpha+1 ; 1,1, i, p},
\end{aligned}
$$

that vanishes on $\boldsymbol{\Delta}=\mathbf{0}$.

\section{Third order Lie remarkable equations}

Third order Lie remarkable equations characterized by the Lie algebra of affine transformations, due to dimensionality considerations, exist for $n=2$ and $m=1$, where the third order jet space and the Lie algebra have the same dimension. In fact, in [22], the following third order Lie remarkable differential equation has been characterized:

$$
\begin{aligned}
& u_{, 11}^{3} u_{, 222}^{2}+u_{, 22}^{3} u_{, 111}^{2}+6 u_{, 11} u_{, 12} u_{, 22} u_{, 111} u_{, 222}-6 u_{, 12} u_{, 22}^{2} u_{, 111} u_{, 112} \\
& \quad-6 u_{, 11} u_{, 22}^{2} u_{, 111} u_{, 122}-6 u_{, 11}^{2} u_{, 12} u_{, 122} u_{, 222}-6 u_{, 11}^{2} u_{, 22} u_{, 112} u_{, 222} \\
& \quad-8 u_{, 12}^{3} u_{, 111} u_{, 222}+9 u_{, 11} u_{, 22}^{2} u_{, 112}^{2}+9 u_{, 11}^{2} u_{, 22} u_{, 122}^{2}+12 u_{, 12}^{2} u_{, 22} u_{, 111} u_{, 122} \\
& +12 u_{, 11} u_{, 12}^{2} u_{, 112} u_{, 222}-18 u_{, 11} u_{, 12} u_{, 22} u_{, 112} u_{, 122}=0 .
\end{aligned}
$$


Equation (27) admits also the symmetries generated by

$$
a\left(x_{1} \frac{\partial}{\partial x_{1}}+x_{2} \frac{\partial}{\partial x_{2}}+u \frac{\partial}{\partial u}\right), \quad a \in\left\{x_{1}, x_{2}, u\right\},
$$

i.e., it is invariant with respect to the Lie algebra of projective transformations of $\mathbb{R}^{3}$.

For $n=3$ and $m=1$, the dimension of Lie algebra of affine transformations is 20, whereas the dimension of third order jet space is 23 , so that we can not characterize a third order Lie remarkable equation. Even if we take the Lie algebra of projective transformations (with dimension 24), neither strongly nor weakly Lie remarkable equations exist, since the rank of the distribution generated by the third order prolongation of vector fields of projective transformations is 20 .

For $n=2$ the dimension of the Lie algebra of projective transformations, $d=(m+2)(m+4)$, is greater than the dimension of the jet space, $j=$ $10 m+2$ for all values of $m>0$, so that we may try to construct third order strongly Lie remarkable equations involving two independent variables and an arbitrary number of dependent ones. Thus, in principle, we may conjecture that a hierarchy of Lie remarkable third order systems involving the unknowns $u_{\alpha}\left(x_{1}, x_{2}\right)(\alpha=1, \ldots, m, m \geq 2)$ may exist.

Taking $m=2$, the computation can be easily done, and the following result can be stated. 
Theorem 10 The third order system of partial differential equations

$$
\begin{aligned}
& \left(u_{1,22} u_{2,12}-u_{1,12} u_{2,22}\right)\left(\left(u_{1,22} u_{2,11}-u_{1,12} u_{2,12}\right)^{2}\right. \\
& \left.\quad-\left(u_{1,11} u_{1,22}-u_{1,12}^{2}\right)\left(u_{2,11} u_{2,22}-u_{2,12}^{2}\right)\right) u_{1,111} \\
& +3\left(u_{1,11} u_{2,12}-u_{1,12} u_{2,11}\right)\left(u_{1,12} u_{2,12}-u_{1,22} u_{2,11}\right) \times \\
& \quad \times\left(u_{1,12} u_{2,22}-u_{1,22} u_{2,12}\right) u_{1,112} \\
& +3\left(u_{1,11} u_{2,12}-u_{1,12} u_{2,11}\right)^{2}\left(u_{1,22} u_{2,12}-u_{1,12} u_{2,22}\right) u_{1,122} \\
& +\left(u_{1,11} u_{2,12}-u_{1,12} u_{2,11}\right)^{2}\left(u_{1,11} u_{2,22}-u_{1,12} u_{2,12}\right) u_{1,222} \\
& +\left(u_{1,11} u_{1,22}-u_{1,12}^{2}\right)\left(u_{1,22} u_{2,11}-u_{1,11} u_{2,22}\right)\left(u_{1,12} u_{2,22}-u_{1,22} u_{2,12}\right) u_{2,111} \\
& +3\left(u_{1,12}^{2}-u_{1,11} u_{1,22}\right)\left(u_{1,12} u_{2,11}-u_{1,11} u_{2,12}\right)\left(u_{1,12} u_{2,22}-u_{1,22} u_{2,12}\right) u_{2,112} \\
& +\left(u_{1,12}^{2}-u_{1,11} u_{1,22}\right)\left(u_{1,12} u_{2,11}-u_{1,11} u_{2,12}\right)^{2} u_{2,222}=0, \\
& 3\left(u_{1,22} u_{2,12}-u_{1,12} u_{2,22}\right)^{2}\left(u_{2,11} u_{2,22}-u_{2,12}^{2}\right) u_{1,112} \\
& +3\left(u_{1,11} u_{2,22}-u_{1,22} u_{2,11}\right)\left(u_{1,22} u_{2,12}-u_{1,12} u_{2,22}\right)\left(u_{2,11} u_{2,22}-u_{2,12}^{2}\right) u_{1,122} \\
& +\left(u_{2,11} u_{2,22}-u_{2,12}^{2}\right)\left(\left(u_{1,11} u_{2,22}-u_{1,22} u_{2,11}\right)^{2}\right. \\
& \left.+\left(u_{1,12} u_{2,22}-u_{1,22} u_{2,12}\right)\left(u_{1,12} u_{2,11}-u_{1,11} u_{2,12}\right)\right) u_{1,222} \\
& +\left(u_{1,22} u_{2,12}-u_{1,12} u_{2,22}\right)^{3} u_{2,111} \\
& +3\left(u_{1,12} u_{2,12}-u_{1,22} u_{2,11}\right)\left(u_{1,22} u_{2,12}-u_{1,12} u_{2,22}\right)^{2} u_{2,112} \\
& + \\
& +3\left(u_{1,22} u_{2,12}-u_{1,12} u_{2,22}\right)\left(\left(u_{1,22} u_{2,11}-u_{1,12} u_{2,12}\right)^{2}\right. \\
& + \\
& \left.+\left(u_{1,11} u_{1,22}-u_{1,12}^{2}\right)\left(u_{2,11} u_{2,22}-u_{2,12}^{2}\right)\right) u_{2,122} \\
& + \\
& +\left(\left(u_{1,12} u_{2,12}-u_{1,22} u_{2,11}\right)^{3}-\left(u_{1,11} u_{2,22}+u_{1,12} u_{2,12}-2 u_{1,22} u_{2,11}\right) \times\right. \\
& \left.\quad \times\left(u_{1,11} u_{1,22}-u_{1,12}^{2}\right)\left(u_{2,11} u_{2,22}-u_{2,12}^{2}\right)\right) u_{2,222}=0 \\
&
\end{aligned}
$$

characterizing a 20-dimensional submanifold in $J^{3}\left(\mathbb{R}^{4}, 2\right)$, is strongly Lie remarkable with respect to the Lie algebra of projective transformations of $\mathbb{R}^{4}$.

Proof. In fact, the rank of the distribution of the third order prolongations of the vector fields of Lie algebra of projective transformations of $\mathbb{R}^{4}$ is maximal (and equal to the dimension of third order jet space). Moreover, the rank lowers to 20 when evaluated on the submanifold described by (29), and this completes the proof.

For higher values of $m$, the amount of computation required to look for possible third order strongly Lie remarkable equations is rapidly increasing. Work in this direction is in progress. 


\section{Conclusions}

In this paper, we derived some new second and third order partial differential equations uniquely determined by the Lie algebra of affine or projective transformations of $\mathbb{R}^{n+m}$. The characterization of second order partial differential equations is done by using only the Lie algebra of affine transformations; however, we proved that these equations are also invariant with respect to the projective transformations. Affine transformations are sufficient to characterize a third order scalar strongly Lie remarkable equation in two independent variables, whereas for a system of two third order differential equations in two independent variables we need all the symmetries in the Lie algebra of projective transformations.

As far as the second order strongly Lie remarkable differential equations here identified are concerned, by using the property according to which they are in one-to-one correspondence with the Lie algebra $\mathcal{P}\left(\mathbb{R}^{n+m}\right)$ of projective transformations, as the classical Monge-Ampère equation for a surface in $\mathbb{R}^{3}$ with vanishing Gaussian curvature, we can consider them as the instances of a hierarchy of second order Monge-Ampère equations in $\mathbb{R}^{n+m}$. Recalling also that the simplest second order scalar ordinary differential equation is invariant with respect to the 8-dimensional Lie algebra of projective transformations of the plane, the hierarchy of second order differential equations reads:

$$
\begin{array}{ll}
\frac{d^{2} u}{d x^{2}}=0, & \mathcal{P}\left(\mathbb{R}^{2}\right), \\
\frac{\partial^{2} u}{\partial x_{1}^{2}} \frac{\partial^{2} u}{\partial x_{2}^{2}}-\left(\frac{\partial^{2} u}{\partial x_{1} \partial x_{2}}\right)^{2}=0, & \mathcal{P}\left(\mathbb{R}^{3}\right), \\
\operatorname{det}\left(\left\|\frac{\partial^{2} u}{\partial x_{i} \partial x_{j}}\right\|\right)=0, & \mathcal{P}\left(\mathbb{R}^{n+1}\right), \\
\frac{\partial^{2} u_{\alpha}}{\partial x_{1}^{2}} \frac{\partial^{2} u_{\alpha+1}}{\partial x_{p} \partial x_{q}}-\frac{\partial^{2} u_{\alpha+1}}{\partial x_{1}^{2}} \frac{\partial^{2} u_{\alpha}}{\partial x_{p} \partial x_{q}}=0, & \mathcal{P}\left(\mathbb{R}^{n+m}\right),
\end{array}
$$

where $\alpha=1, \ldots, m-1$ and $i, j, p, q=1, \ldots, n$.

Lie remarkable systems of partial differential equations determined by other relevant Lie algebras of point symmetries are currently investigated and will be the object of a forthcoming paper. 


\section{Acknowledgments}

This research has been partly supported by G.N.F.M. of "Istituto Nazionale di Alta Matematica".

\section{References}

[1] L. V. Ovsiannikov, Group Analysis of Differential Equations, Academic Press, New York, 1982.

[2] N. H. Ibragimov, Transformation Groups Applied to Mathematical Physics, D. Reidel Publishing Company, Dordrecht, 1985.

[3] P. J. Olver, Applications of Lie Groups to Differential Equations, Second edition, Springer, 1991.

[4] P. J. Olver, Equivalence, Invariants, and Symmetry, Cambridge University Press, New York, 1995.

[5] V. Bocharov, V. N. Chetverikov, S. V. Duzhin, N. G. Khor'kova, I. S. Krasil'shchik, A. V. Samokhin, Yu. N. Torkhov, A. M. Verbovetsky, A. M. Vinogradov, Symmetries and Conservation Laws for Differential Equations of Mathematical Physics. I. S. Krasil'shchik, A. M. Vinogradov eds., Translations of Math. Monographs, 182, AMS, 1999.

[6] G. Baumann, Symmetry Analysis of Differential Equations with Mathematica, Springer, Berlin, 2000.

[7] G. W. Bluman, S. C. Anco, Symmetry and Integration Methods for Differential Equations, Springer, New York, 2002,

[8] G. W. Bluman, A. F. Cheviakov, S. C. Anco, Applications of Symmetry Methods to Partial Differential Equations, Springer, New York, 2009.

[9] G. W. Bluman, J. D. Cole, Similarity Methods for Differential Equations, Springer-Verlag, New York, 1974.

[10] V. Rosenhaus, On one-to-one correspondence between the equation and its group. The Monge-Ampère equation, Preprint F. 18 Acad. Sci. Estonian SSR-Tartu (1982) 1-16. 
[11] V. Rosenhaus, The unique determination of the equation by its invariance group and field-space symmetry, Algebras Groups Geom. 3 (1986) 148-166.

[12] V. Rosenhaus, Groups of invariance and solutions of equations determined by them, Algebras Groups Geom. 5 (1988) 137-150.

[13] J. Krause, On the complete symmetry group of the classical Kepler system, J. Math. Phys. 35 (1994) 5734-5748.

[14] M. C. Nucci, The complete Kepler group can be derived by Lie group analysis, J. Math. Phys. 37 (1996) 1772-1775.

[15] K. Andriopoulos, P. G. L. Leach, G. P. Flessas, Complete symmetry groups of ordinary differential equations and their integrals: Some basic considerations, J. Math. Anal. Appl. 262 (2001) 256-273.

[16] S. M. Myeni, P. G. L. Leach, Heuristic analysis of the complete symmetry group and nonlocal symmetries for some nonlinear evolution equations, Math. Meth. Appl. Sci. 30 (2007) 2065-2077.

[17] W. I. Fushchych, I. Yehorchenko, Second-order differential invariants of the rotation group $\mathrm{O}(\mathrm{n})$ and of its extension: $\mathrm{E}(\mathrm{n}), \mathrm{P}(1, \mathrm{n}), \mathrm{G}(1, \mathrm{n})$, Acta App. Math. 28 (1992) 69-92.

[18] F. Oliveri, Lie symmetries of differential equations: direct and inverse problems, Note di Matematica 23 (2005) 195-216.

[19] F. Oliveri, Sur une proprieté remarquable des équations de MongeAmpère, Rend. Circ. Mat. Palermo, Supplemento 78 Ser. II (2006) 243257.

[20] G. Manno, F. Oliveri, R. Vitolo, On an inverse problem in group analysis of PDEs: Lie-remarkable equations, Proc. "Wascom 2005" (Editors: R. Monaco, G. Mulone, S. Rionero, T. Ruggeri), World Scientific, Singapore (2006) 420-431.

[21] G. Manno, F. Oliveri, R. Vitolo, On differential equations characterized by their Lie point symmetries, J. Math. Anal. Appl. 332 (2007) 767-786. 
[22] G. Manno, F. Oliveri, R. Vitolo, Differential equations uniquely determined by algebras of point symmetries, Theor. Math. Phys. 151 (2007) 843-850.

[23] G. Manno, F. Oliveri, R. Vitolo, On the correspondence between differential equations and symmetry algebras. In Symmetry and Perturbation Theory, Proc. Int. Conf. SPT 2007; G. Gaeta, R. Vitolo, S. Walcher, Eds; World Scientific, Singapore (2007) 164-171.

[24] G. Manno, F. Oliveri, G. Saccomandi, R. Vitolo, Ordinary differential equations described by their Lie symmetry algebra, J. Geom. Phys. 85 (2014) 2-15.

[25] A. C. Hearn, Reduce User's Manual, Santa Monica, CA, USA, 2004.

[26] F. Oliveri, ReLie: A Reduce program for Lie group analysis of differential equations, Submitted (2019).

[27] A-M. Ampère, Considérations générales sur les intégrales des équations aux différences partielles, Journal de l'École Polytechnique 10 (1815) 549-611.

[28] G. Boillat, Le champ scalaire de Monge-Ampère, Det Kgl. Norske Vid. Selsk. Forth. 41 (1968) 78-81.

[29] T. Ruggeri, Su una naturale estensione a tre variabili dell'equazione di Monge-Ampère, Rend. Accad. Naz. Lincei 55 (1973) 445-449.

[30] A. Donato, U. Ramgulam, C. Rogers, The $(3+1)$-dimensional MongeAmpère equation in discontinuity were theory: Application of a reciprocal transformation, Meccanica 27 (1992) 257-262.

[31] G. Boillat, Sur l'équation générale de Monge-Ampère à plusieurs variables, C. R. Acad. Sci. Paris 313 (1991) 805-808.

[32] F. Oliveri, Linearizable second order Monge-Ampère equations, J. Math. Anal. Appl. 218 (1998) 329-345.

[33] M. Gorgone, F. Oliveri, Nonlinear first order partial differential equations reducible to first order homogeneous and autonomous quasilinear ones, Ricerche di Matematica 66 (2017) 51-63. 
[34] M. Gorgone, F. Oliveri, Nonlinear first order PDEs reducible to autonomous form polynomially homogeneous in the derivatives, J. Geom. Phys. 113 (2017) 53-64.

[35] G. Boillat, Sur l'équation générale de Monge-Ampère d'ordre supérieur, C. R. Acad. Sci. Paris Sér. I. Math. 315 (1992) 1211-1214.

[36] G. Ganchev, V. Milousheva, On the theory of surfaces in the fourdimensional Euclidean space, Kodai Math. J. 31 (2008) 183-198. 